\title{
Graph Colouring for Office Blocks $\dagger$
}

\author{
David Allwright ${ }^{1} \ddagger$, Tim Gould ${ }^{2}$, Jens Gravesen $^{3}$, Robert Leese $^{1}$, \\ and Henrik G. Petersen ${ }^{4}$ \\ ${ }^{1}$ Smith Institute \\ ${ }^{2}$ Lancaster University \\ ${ }^{3}$ Technical University of Denmark \\ ${ }^{4}$ University of Southern Denmark \\ (Communicated to MIIR on 27 July 2021)
}

Study Group: ESGI 53. Manchester, UK, 21-24 March 2005.

Communicated by: Hilary Ockendon

Industrial Partner: BAE Ssystems ATC, Filton

Presenter: Colin Sillence

Team Members: David Allwright, Smith Insitute; Tim Gould, Lancaster University; Jens Gravesen, Technical University of Denmark; Robert Leese, Smith Insitute; Henrik G. Petersen, University of Southern Denmark; Arash Rafiey, Royal Holloway, London; Bjarne Toft, University of Southern Denmark; Eddie Wilson, University of Bristol.

Industrial Sector: Aerospace

Tools: Graph colouring

Key Words: WLANs, electromagnetic screening, wireless communications, graph colouring

MSC2020 Codes: 20, 94

\section{Summary}

How can different organizations in the same building each maintain security of their internal wifi communications? Part of the answer to this is for organizations on opposite sides of a single wall or floor to use distinct radio channels. How many channels may be needed to meet this requirement in a typical modern office building? Mathematically this channel assignment problem is a vertex colouring problem with the organizations as the vertices, joined if somewhere they are adjacent either wall-to-wall or floor-to-ceiling. The study group showed that there is no easy answer to this question, but that bounds can be obtained in particular cases. For instance if each company only occupies one floor of the building, 8 channels suffice. Public graph-colouring algorithms are quick and successful in finding minimal colourings for reasonably complex building layouts.

$\dagger$ The work mentioned at the end of Section 2.5 is published as: Painting the Office. Bruce Reed, David Allwright. Mathematics-in-Industry Case Studies Journal, Volume 1, pp. 1-8 (2008).

$\ddagger$ Corresponding author. Email: allwrigh@maths.ox.ac.uk 


\section{Introduction}

The use of Wireless Local Area Networks (WLANs) is increasing, but raises numerous problems of security. BAE Systems, working for OFCOM has recently demonstrated a technology (unhelpfully dubbed 'Stealthy Wallpaper') that could address some of these problems. The technology is an application of adaptive or active Frequency Selective Surfaces (FSS) which for the purposes of this discussion may be considered as thin planar tunable band-pass filters.

Consider for example a large office complex with space rented to numerous independent organisations. WLANs operating on any frequencies could be used with total security provided electromagnetic screening of over 100dBs was implemented around each organisation's territory. This is impractical both on the grounds of expense and inflexibility as organisations grow, shrink, die, or are initiated. Additionally mobile 'phone communication across boundaries would be precluded, which would have safety and other implications.

However, it would not be prohibitively expensive to build or retro-fit moderate screening (say 55-75 dB) between each office, and include small (about $0.3 \mathrm{~m}^{2}$ ) windows of 'stealthy wallpaper' which can be switched by a central 'controller' to pass specified frequencies of WLAN channels or to be opaque to all such frequencies. (They could be designed to simultaneously pass mobile 'phone frequencies.)

This degree of screening would not be sufficient to guarantee blocking of WLAN channels between adjacent offices, but it would be enough to guarantee blocking across two boundaries. We are therefore interested in forcing adjacent territories to be on different WLAN channels. (Consider territories as adjacent if and only if they share an area of wall floor or ceiling. Territories 'meeting' at a line or point need not be considered adjacent.) This gives rise to the following graph colouring problems.

\subsection{Mathematical Problem}

The set of possible allocations of office space in a multi-storey office complex is an (as-yet) ill-defined subset of the set of all possible arrangements of touching bounded volumes in $\mathbb{R}^{3}$. Atria, stairwells, and lift shafts need to be considered. We would like to know several things:

(1) Can the class of adjacency graphs produced by arrangements of office territories be described mathematically?

(2) What is the maximum chromatic number of this class of adjacency graphs?

(3) If not (1), then can a 'layman-interpretable' restriction be placed on the allowable configurations of office territory in order to yield a class of adjacency graphs with known maximum chromatic number (or a reasonably tight upper bound $U$ for the maximum chromatic number). The restriction should be as weak as possible, i.e. ideally only rule out extreme or unlikely allocations. The chromatic number 
(upper bound) should be as small as possible. There will be a trade-off between the strength of the restriction and the maximum chromatic number, which may be worth investigating.

(4) Is there an algorithm to produce a colouring using $U$ colours or fewer for any given adjacency graph in the class? The efficiency of this algorithm is not usually of great concern, but may get problematic when the number of nodes get large. For practical purposes assume the number of nodes to be 100 or fewer. As a guide, the algorithm should sort out the colouring in a day or less on a modern PC.

\section{Results}

The short and simple answer to the problem proposed by BAE is that no reasonable upper bound to the colouring of offices can be proposed. This is because the colouring of $\mathbb{R}^{3}$ is an unbounded problem. By way of example, let us consider regions formed by the connected union of two Cartesian cuboids. An example going back to Tietze [1] (and probably earlier) shows that we can have $n$ regions all in contact with each other. For we can consider a 2-storey cuboidal building whose ground floor consists of $n$ corridors running North-South, and whose first floor consists of $n$ corridors running East-West. Let each territory be the union of one corridor on the ground floor and one on the first floor. Then each territory is a connected union of two Cartesian cuboids, and any pair of territories are in contact, so the clique number and the chromatic number are both $n$. (We recall the definitions: the clique number of a graph is the size of the largest clique, i.e. the largest set of nodes that are all joined to each other, and is sometimes denoted $\omega(G)$ or $\operatorname{cl}(G)$. In our context, this is the maximum number of territories that are all in face-to-face contact with each other. The chromatic number of a graph is the smallest number of colours needed to colour the nodes in such a way that any pair of joined nodes are distinct colours, generally denoted $\chi(G)$. In our context, this is the minimum number of frequencies needed to assign one to each territory in such a way that territories in face-to-face contact have distinct frequencies. Since all the nodes in a clique must have different colours, the chromatic number is always at least as large as the clique number: $\chi(G) \geq \omega(G)$.)

While the general problem cannot be solved, we can obtain results for a number of specific layouts of office blocks. Furthermore we have designed an algorithm (presented in section 3) which should obtain a good colouring for all but the most stubborn cases.

We present here some case for which an upper bound can easily be obtained. That is, denoting the optimal number of colours of a given type of office as $\chi$, we can find an upper bound for $\chi$. Unfortunately, none of these known cases are practical in a real office building and they are presented here more for completeness than practical use. We should take note here that in our office buildings, we would always use one extra colour for shared spaces. This is ignored when we present our results to keep a simple agreement with standard colouring problems.

The known cases we present here are: 
(1) Planar office building

(2) Buildings with only single floor territories

(3) Office buildings with concentric rings separated by hallways

(4) Offices with only box shaped offices.

Furthermore there are two simple but impractical bounds which we will briefly present here.

\subsection{Simple bounds}

The first bound we will present is a trivial one. If a building has a total number of territories $T$ then $\chi \leq T$. Since we cannot choose a colouring with more than $T$ colours, this bound is proved.

The second we present is less trivial, but unfortunately not much more useful: the upper bound on the colouring $\chi \leq M+1$ where $M$ is the maximum number of neighbours of any of the territories (the maximum vertex-degree of the graph). This a form of Brooks' theorem which can be found in [2] for example. (If $M+1$ colours are available then you can colour the nodes one-by-one and there is always a free colour available.)

\subsection{Planar Office Block}

In the case of a planar office, the four colour theorem (FCT) implies that $\chi \leq 4$. The FCT was first presented in [3, 4] and more recently refined in [5] and states that any planar graph can be coloured in four or less colours.

Since we will be revisiting the FCT later in this report, we note here that an algorithm exists to find a four colouring of a planar graph in quadratic time [5]. This is an important result as typically a colouring problem is NP-complete.

\subsection{Single-Storey Offices Only}

When an office contains only offices with a single storey, we can consider each floor to be a separate planar office. This guarantees us an upper bound $\chi \leq 8$ to the colouring.

Proof that an 8 colouring exists. Let us look at each storey individually. Since no offices cross territories, we do not have to worry about the connection between storeys. Colouring each odd numbered storey with one set of four colours (as in section 2.2) and each even storey with a different and unique set of four colours guarantees that no vertical neighbours can share a colour (as they are guaranteed to be coloured from a different set). As such $\chi \leq 8$. 
While typically, the number of colours required for the alternating storeys will be less than eight, figure 2.3 shows a two storey office block which requires all eight colours, and shows that 8 are still necessary even if the offices are required to be convex.

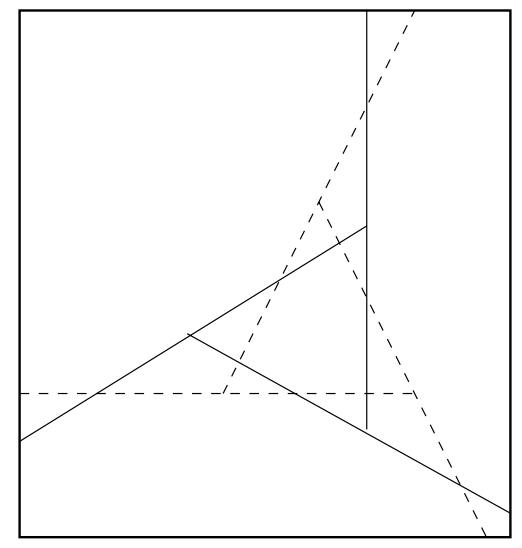

Figure 1: A two-storey square building. The ground floor is divided into 4 convex rooms by the solid lines, and the first floor similarly by the dashed lines. Each of the resulting 8 rooms is in face-to-face contact with all the others.

\subsection{Concentric Rings}

Sometimes the geometry of a building may give a floor plan which looks like a series of concentric rings of offices separated by halls and other public spaces. In such an office $\chi \leq 4$ as the office can be considered as a set of unconnected (due to the public spaces) planar rings. Each ring can then be four coloured, but we may use the same colours for each ring (as opposed to the previous case).

\subsection{Box-shaped Office Territories}

A simple (but impractical) restriction to put on office territories is to assume they are box-shaped, i.e. Cartesian cuboids all aligned with a fixed set of axes. The hope is that such a configuration has a low chromatic number.

We can make a few observations

(1) The minimal degree is unbounded, cf. Figure 2.

(2) The clique number is bounded by 4 .

Proof of 2. Consider a box configuration where each pair of boxes are neighbours. As two boxes with a third box in between can't be neighbours, a given box can have neighbours on at most three sides. So we have the three cases depicted in Figure 3. 


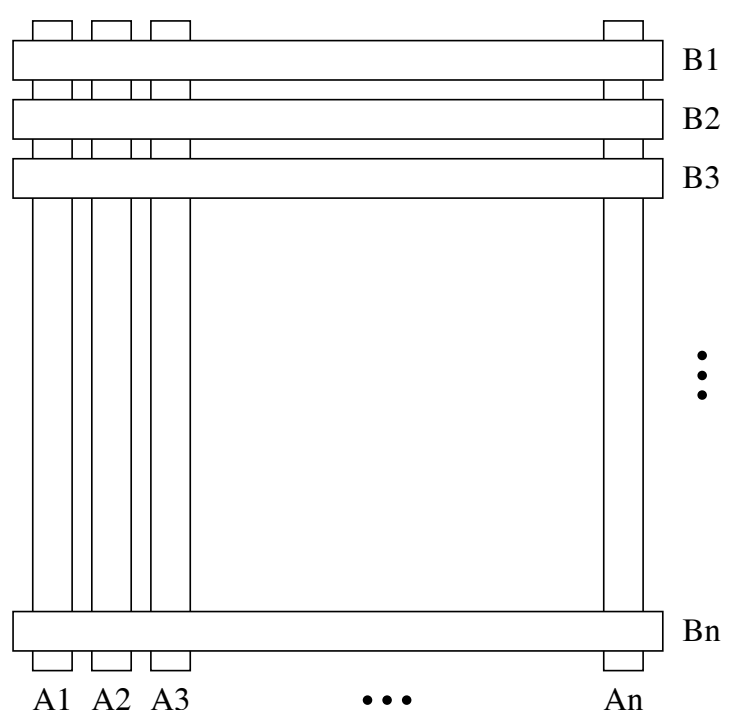

Figure 2: A configuration of box-shaped territories with minimal degree $n$.
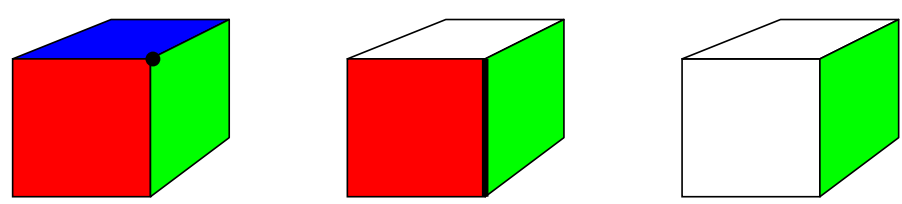

Figure 3: The three possible cases.

In case 1 the given box has neighbours on both the red, green, and blue side. As a neighbour on the green side is a neighbour to the ones on both the blue and red side it has to touch the common corner of the three coloured sides. So there can be only one neighbour on the green side and similarly on the red and blue side. All in all we can have at most four boxes in the configuration.

In the second case the given box has neighbours on the red and green side. As a neighbour on the green side is a neighbour to the ones on the red side it has to touch the line between the red and green side. Furthermore, the neighbours on the green side touch each other pairwise so there can be at most two neighbours on the green side and similarly on the red side. If we have only one neighbour on the green side we have at most four boxes all in all.

So now assume we have two neighbours on both the red and green sides. Both of the two neighbours on the green side touch the line between the red and green side and each other so they touch a single common point on the line between the red and green side. A neighbour on the red side also has to touch this point so if we cut the all the neighbours along the line between the red and green side we get the picture in Figure 4. A side of the top red box overlaps a side of the lower green box so they have to be extended across the line between the red and green side. The same argument holds for the lower red box and the upper green box, but we can't extend all four boxes so on one of the sides there can only be one box and we have at most four boxes all in all.

We finally come to the third case where the given box has neighbours on only the green 


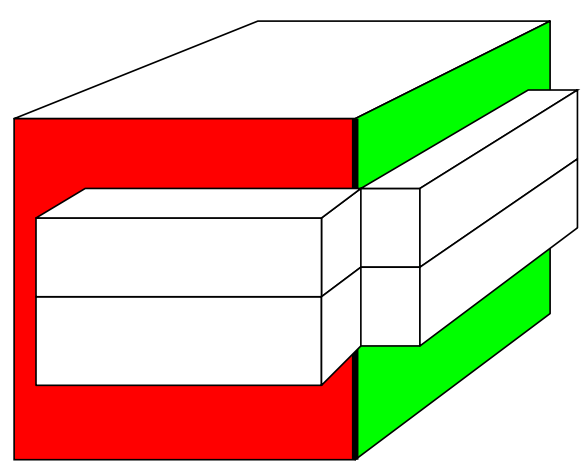

Figure 4: We can not make all four boxes touch pairwise.

side. The problem is now reduced to the case of rectangles in the plane where we can have only three which touches each other pairwise. So once more we have at most four boxes.

The unbounded minimal degree suggests that the chromatic number might be unbounded, while the low clique-number suggest that we have a low chromatic number too.

It is not difficult to make examples with chromatic number 5 , and a chromatic number of 6 is attained, for instance, for the example shown in Figure 2.5. Finding a 6-colouring is straightforward, but if we try to colour it in 5 colours, then without loss of generality $A, B$ and $D$ are coloured 1,2 and 3 respectively. Then $F$ and $G$ cannot be coloured 1 or 2 , and cannot both be coloured 3 , so by the left-right symmetry of the arrangement, we may assume that $F$ is coloured 4. Then $E$ is touching $A, B, D, F$ and so must be coloured 5. $I$ is touching $A, D, E, F$ and so must be coloured 2. $H$ is touching $B, D, E, F$ and so must be coloured 1 . But then $J$ is touching $D, E, F, H, I$ which use up all 5 colours.

We have in collaboration with Carsten Thomassen tried to bound the chromatic number but so far without success. ${ }^{1}$

\subsection{Convex volumes}

If we allow the regions to be general convex volumes rather than Cartesian cuboids, then still the clique number can be arbitrarily large. We are grateful to Ross Kang for drawing this result to our attention. The problem was apparently first raised by Paul Stäckel in 1899, and solved by Tietze [6], then posed independently by Crum and solved by Besicovitch [7], and another solution is by Dewdney and Vranch [8], who showed that the Voronoi volumes of any $n$ distinct points on the twisted cubic all have face-to-face contact.

\footnotetext{
${ }^{1}$ Since the Study Group, Bruce Reed and Adrian Vetta have discovered an ingenious construction showing that the chromatic number is actually unbounded, and can be at least a constant times $\log \log n / \log \log \log n$ (where $n$ is the number of boxes). We are very grateful to Ross Kang for bringing this to our attention.
} 

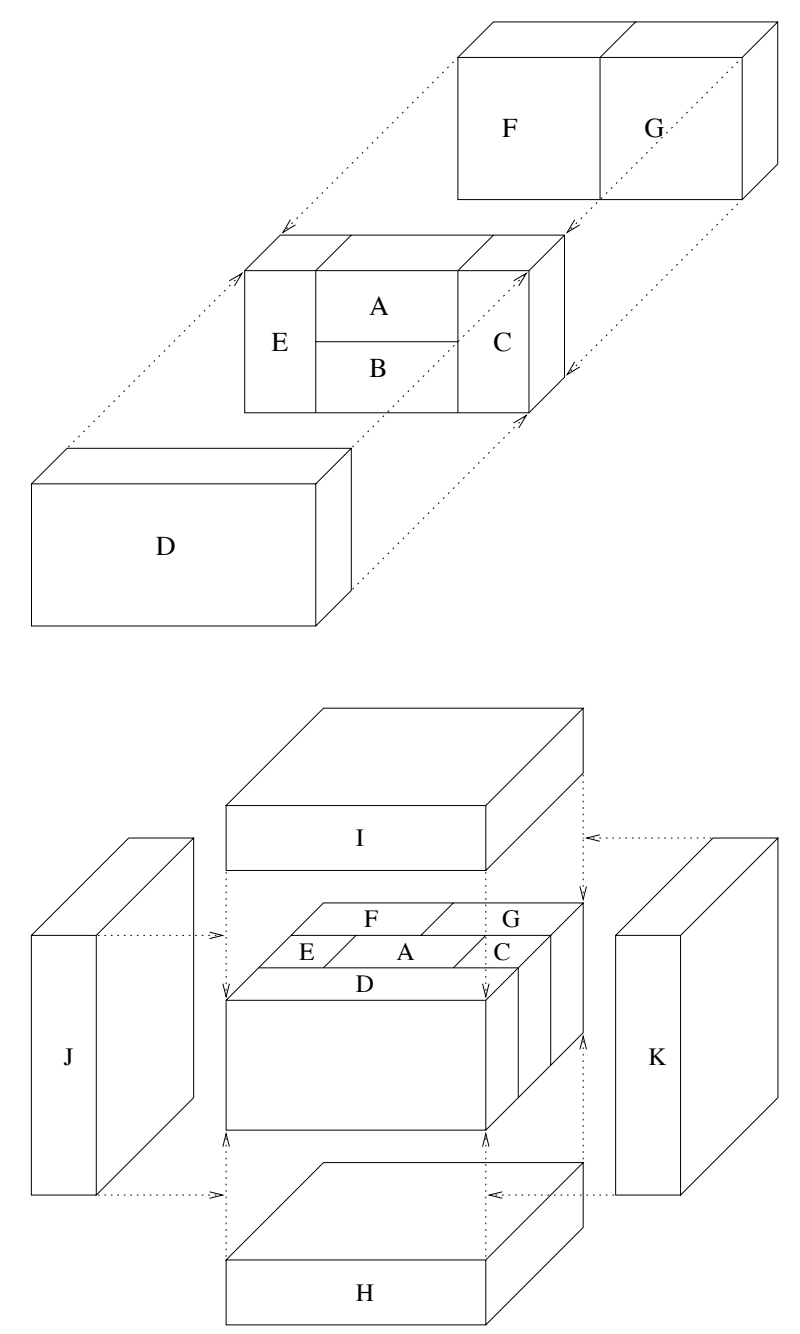

Figure 5: Diagrammatic construction of a set of 11 Cartesian cuboids that need 6 colours if every pair of touching cuboids are to be different colours. First cuboids $\mathrm{A}-\mathrm{G}$ are assembled as in the top diagram, and then $\mathrm{H}-\mathrm{K}$ are added as below.

Besicovitch's solution essentially arises from observing that $n$ pieces of paper in the plane can be arranged to all overlap each other (as illustrated for $n=4$ in Figure 6 ) and then showing that the pieces of paper can be expanded up into thin convex polyhedral wedges all in contact with each other.

For the construction by Voronoi volumes, consider $n$ points $P_{1}, P_{2}, \ldots, P_{n}$ and let

$$
V_{i}=\left\{\mathbf{x}:\left|\mathbf{x}-P_{i}\right| \leq\left|\mathbf{x}-P_{j}\right| \text { for all } j \neq i\right\},
$$

the Voronoi volume. This is clearly convex and polyhedral, and the crucial thing is to choose the $P_{i}$ in such a way that each pair $V_{i}, V_{j}$ have a common face. This face, if it exists, must consist of points $\mathbf{x}$ such that

$$
\left|\mathbf{x}-P_{i}\right|=\left|\mathbf{x}-P_{j}\right|<\left|\mathbf{x}-P_{k}\right| \text { for all } k \neq i, j .
$$




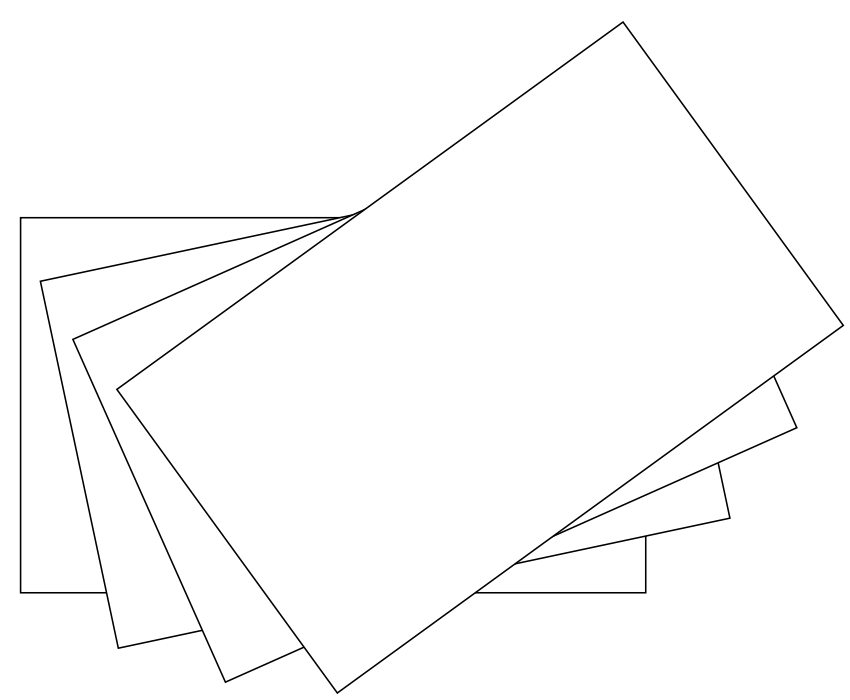

Figure 6: $n$ pairwise-overlapping pieces of paper, illustrated for $n=4$.

This face is therefore an area of the plane $\Pi_{i j}$ that perpendicularly bisects $P_{i} P_{j}$. In fact, if we can show that there is a single point $\mathbf{x}$ satisfying (2), that will suffice, since at least a small area of $\Pi_{i j}$ around $\mathbf{x}$ will then still satisfy (2) and so form part of the common face.

We shall show that such an $\mathbf{x}$ exists if $P_{1}, P_{2}, \ldots, P_{n}$ are chosen as distinct points on any curve $C$ with the following property: for any distinct points $P, Q$ on $C$ there is a sphere, tangent to $C$ at $P$ and $Q$, and with the rest of $C$ lying strictly outside the sphere. Certainly if this property holds, then taking $P$ and $Q$ to be $P_{i}$ and $P_{j}$, the centre of the sphere is a point $\mathbf{x}$ with the required property (2), since $\left|\mathbf{x}-P_{i}\right|=\left|\mathbf{x}-P_{j}\right|=R$, the radius of the sphere, and since all the other $P_{k}$ lie on the curve they are strictly outside the sphere, so $\left|\mathbf{x}-P_{k}\right|>R$.

So now it is enough to produce a curve $C$ that has the stated property, and we shall show that the twisted cubic $\mathbf{r}(t)=\left(t, t^{2}, t^{3}\right)$ does. Suppose $P=\mathbf{r}(u)$ and $Q=\mathbf{r}(v)$. For a sphere $|\mathbf{r}-\mathbf{a}|^{2}=R^{2}$ to be tangent to $C$ at $P$, its centre a must lie on the plane passing through $P$ perpendicular to the curve $C$ at $P$. The same condition must hold for $Q$, and a must lie on the plane perpendicularly bisecting $P Q$, so

$$
\mathbf{r}^{\prime}(u) \cdot \mathbf{a}=\mathbf{r}^{\prime}(u) \cdot \mathbf{r}(u), \quad \mathbf{r}^{\prime}(v) \cdot \mathbf{a}=\mathbf{r}^{\prime}(v) \cdot \mathbf{r}(v), \quad 2(\mathbf{r}(u)-\mathbf{r}(v)) \cdot \mathbf{a}=|\mathbf{r}(u)|^{2}-|\mathbf{r}(v)|^{2} .
$$

Provided that $\mathbf{r}^{\prime}(u), \mathbf{r}^{\prime}(v)$ and $\mathbf{r}(u)-\mathbf{r}(v)$ are linearly independent there is a unique solution a to these, and this linear independence is easily checked for the twisted cubic. We then must show that the rest of the curve $C$ lies outside the sphere, and for this we consider

$$
f(t)=|\mathbf{r}(t)-\mathbf{a}|^{2}-R^{2}
$$

This $f$ is a polynomial in $t$, and by the tangency construction it has (at least) double roots at $u$ and $v$. If it had any more real roots (either distinct from $u$ and $v$, or of greater multiplicity at $u$ or $v$ ) then $f$ would have at least 5 real roots (counting multiplicity) and so $f^{\prime \prime \prime \prime \prime}$ would have at least 1 real root. But from (4)

$$
f^{\prime \prime \prime \prime}(t)=2(\mathbf{r}(t)-\mathbf{a}) \cdot \mathbf{r}^{\prime \prime \prime \prime}(t)+8 \mathbf{r}^{\prime}(t) \cdot \mathbf{r}^{\prime \prime \prime}(t)+6\left|\mathbf{r}^{\prime \prime}(t)\right|^{2}=0+8\left(3 t^{2}\right)(6)+6\left(4+36 t^{2}\right)>0 \text {. }
$$


So in fact $f$ has no roots other than the double roots at $u$ and $v$, and since $f(t)>0$ as $t \rightarrow \pm \infty$, we see $f(t)>0$ for all $t \neq u, v$ as required.

If we want the polyhedra $V_{i}$ to be bounded, we can simply constrain them by $\max \left(\left|x_{1}\right|,\left|x_{2}\right|,\left|x_{3}\right|\right) \leq M$, where $M$ is chosen large enough that this cube contains at least part of all the interfaces. Even if $P_{i}$ and $P_{j}$ are close, the interface of $V_{i}$ and $V_{j}$ remains bounded, and in fact, as $P_{j} \rightarrow P_{i}$ the centre a tends to the centre of spherical curvature of $C$ at $P_{i}$.

\section{$3 \quad$ Algorithms and Rules}

Our first step in colouring the office block should be to attempt a good colouring through a known graph colouring algorithm such as that presented in [9]. Unfortunately, while this may work for small office blocks, it is not a polynomial-time algorithm and will fail (by having unacceptably long run time) for larger office blocks. As a rule of thumb, a general office with more than about twenty different territories will be uncolourable in a reasonable time.

To this end, we propose an heuristic algorithm which can give a very quick upper bound $U$ to the colouring and a quick colouring of the offices with $\chi$ colours where $\chi \leq U$. This algorithm should give 'instant' results for most offices (less than one second computing time) to an observer and could potentially be implemented in a control system so that new office layouts can be rapidly trialled to see which is the most efficient.

\subsection{Heuristic Algorithm}

We present here an algorithm which, whilst not guaranteeing the best possible colouring of an office block, should give us a good colouring for all but the most difficult (and unlikely) cases. The idea is to separate out multi-storey territories (MSTs) and treat the rest of the building as containing only single storey territories. The upper bound on its colouring is given by $U=8+$ \#MSTs although we will almost always find a colouring with a smaller colouring. MSTs refer to those office territories which cover at least two different storeys of the office building. While a storey will usually refer to a floor of the real building, sometimes it may be better to rotate the building $90^{\circ}$ if doing so reduces the number of multi-storey offices (the orientation of the lattice make no impact to the employment of the algorithm, but it can certainly make a difference to the efficiency).

For the purpose of this algorithm we let $c_{i}$ represent a colour (where $i=0 . . \infty$ ) and $S_{j}=\left(c_{4 j}, c_{4 j+1}, c_{4 j+2}, c_{4 j+3}\right)$ represent a set of four colours (where $j=0 . . \infty$ ). We will now describe our algorithm.

I Create a subgraph $\mathcal{M}$ of multi-storey territories (MSTs) only (with no connections to single-storey territories).

II Define a graph of the whole building $\mathcal{B}$, where each floor of the MSTs is considered as a separate office so that $\mathcal{B}$ contains no MSTs. 
III Divide $\mathcal{B}$ into independent subgraphs $\mathcal{F}_{k}$ where $\mathcal{F}_{k}$ only contains territories on floor $k$ and is not connected to $\mathcal{F}_{k \pm 1}$.

IV Colour each graph $\mathcal{F}_{k}$ independently using an efficient four-colouring algorithm such as that in [5]. Each floor takes its colours from $S_{k} \bmod 2$ so that the total colouring requires only eight colours $\left(c_{0}, c_{1}, \ldots, c_{7}\right)$.

$\mathrm{V}$ Minimise the number of colours in $\mathcal{M}$ through a heuristic algorithm such as that found in [9]. Often, $\mathcal{M}$ will contain a number of unconnected subgraphs so the total colouring should be quite small. Label each of these colours $c_{8}, c_{9}$ etc.

We now have a quick and efficient colouring of the office block. A pseudo-code example of this algorithm appears in appendix A.

We may wish to stop here if it is an acceptable colouring. However, if we like, we can refine our colouring a little by attempting to reuse in $\mathcal{M}$ the eight colours used to colour $\mathcal{B}$. An outline of how to do this appears below.

First, some definitions. Each MST in $\mathcal{M}$ has a corresponding node in some of the subgraphs $\mathcal{F}_{k}$ which has been assigned a colour from either $S_{0}$ or $S_{1}$ through the fourcolouring in step IV. This follows from our definitions of $\mathcal{F}_{k}$. If we label these nodes $n_{k}^{m}$ and their colours as $c_{k}^{m}$ where $m$ is the label of the node in $\mathcal{M}$ and $k$ is the floor number we can continue our algorithm as follows.

VI Choose some node $m$ of $\mathcal{M}$ and a floor $k$. Choose $k^{\prime} \neq k$, and attempt to recolour $n_{k^{\prime}}^{m}$ with $c_{k}^{n}$ either by redefinition (if $k-k^{\prime} \equiv 1 \bmod 2$ since no colours will be shared between $S_{0}$ and $S_{1}$ ) or by a permutation of the colours in $\mathcal{F}_{k^{\prime}}$ where $c_{k^{\prime}}^{n}=c_{k}^{n}$ but the rest of $S_{k}$ mod 2 are permuted. We now have two options

- If this permutation results in an allowable colouring for the whole building, repeat VI with a new value of $k^{\prime}$.

- Otherwise discard any changes made (ie. leave all the $c_{k}^{m}$ unchanged) and proceed to the next step.

VII Repeat step VI until all $m$ have been either changed or left.

If we are lucky, we should have reduced our total number of colours even further.

\subsection{Design Rules}

In order to make as small an upper bound on the colouring as possible, we list some design rules for the layout of the offices. These refer only to MSTs as it is assumed that the heuristic algorithm given above is to be used. These are listed in order of preference and thus each rule gives a higher upper bound than previous rules. An office designer should try to follow the rules in order. 
Figure 7: An example where a neighbour's neighbour is my neighbour. Here blue (top line) has a neighbour green (bottom right) whose neighbour red (bottom left) is also a neighbour of blue

(1) Try to keep MSTs apart. If no two MSTs are connected by any wall, floor or ceiling then $U=9$.

(2) Avoid situations where an MST's neighbour's neighbour is its neighbour (see figure 7). If these are avoided then $U=10$.

(3) Try to keep MSTs in the corners of the building. This will automatically reduce connections and probably help fulfill (1) and (2).

(4) Avoid 'snakes'. A 'snake' is an office which has separate territories on one or more floors, connected on a different storey. Looking like a $\cap$ or $\cup$ from the side for example.

If the above rules are obeyed, it is reasonable to assume that $U \approx 12$ (because a four colouring of the MSTs probably exists) although this is certainly not a formal condition.

\section{Examples}

Colin Sillence did not provide any actual plans of buildings to work from, but did suggest that Dartington House in Oxford (where OCIAM is housed) would be a good example. It has been used by various organisations at different times and partitioned in different ways, and we are grateful to Keith Gillow for providing us with the floor plans of the 1st, 2nd and 3rd floors, which are shown in Figures 8-10. Some rooms were already labelled in the plans (DH1, DH2 etc), and others we have labelled for our purposes in the figures, so the University Offices Space is rooms UOS1-UOS4, the corridors and cupboards are $\mathrm{C} 1-\mathrm{C} 7$ and so on. The only multi-floor rooms are the stairwells: the east stairwell SE extends to all 3 floors, and the west stairwell SW to floors 1-2. We considered the problem of colouring this with each room regarded as a separate "territory". The floor diagrams are not exact, and in particular may not be exact as regards which rooms are over which parts of other rooms, but we treat them as if they were exact, since this is the nearest we have to representing a real building. A file containing the adjacency lists was constructed by hand, first typing in adjacency lists for each floor, and then for the 1-2 and 2-3 overlaps by overlaying the floor plans on paper and looking at them against a bright light. A short program was written to convert these adjacency lists into a graph description in DIMACS format: the graph has 82 vertices and 280 edges. Then the Smallk algorithm of Joe Culberson [9] was used and it (virtually instantaneously) produces a 5-colouring. Converting that back to the room labels used in the figures, the colouring is as in Table 1. Also, there are a number of cases where 5 rooms all have face-to-face contact (e.g. DH18, DH36, DH25, DH26, DH28), which shows that 5 colours are necessary. 


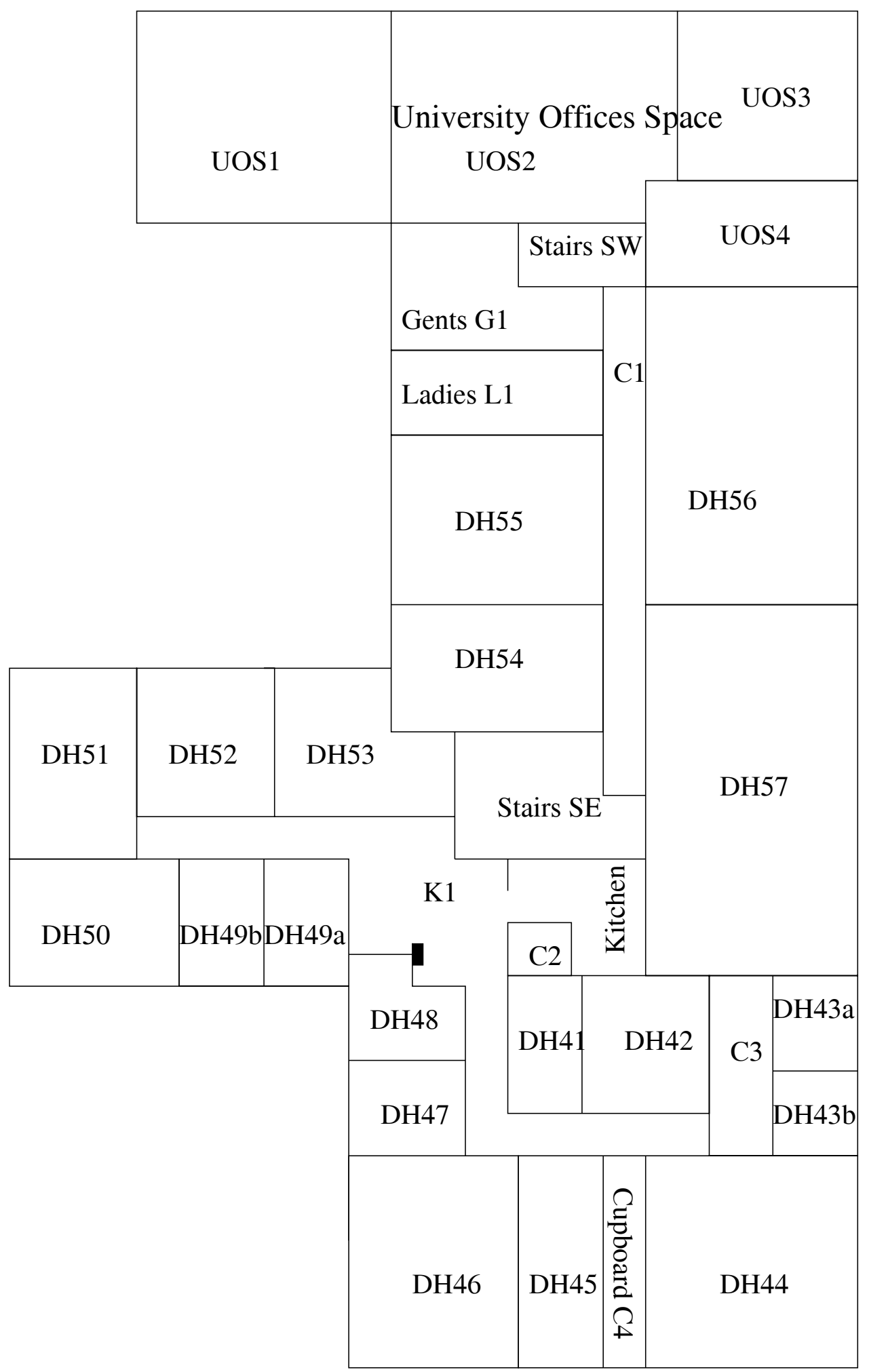

Figure 8: Floor plan of Dartington House 1st floor. 


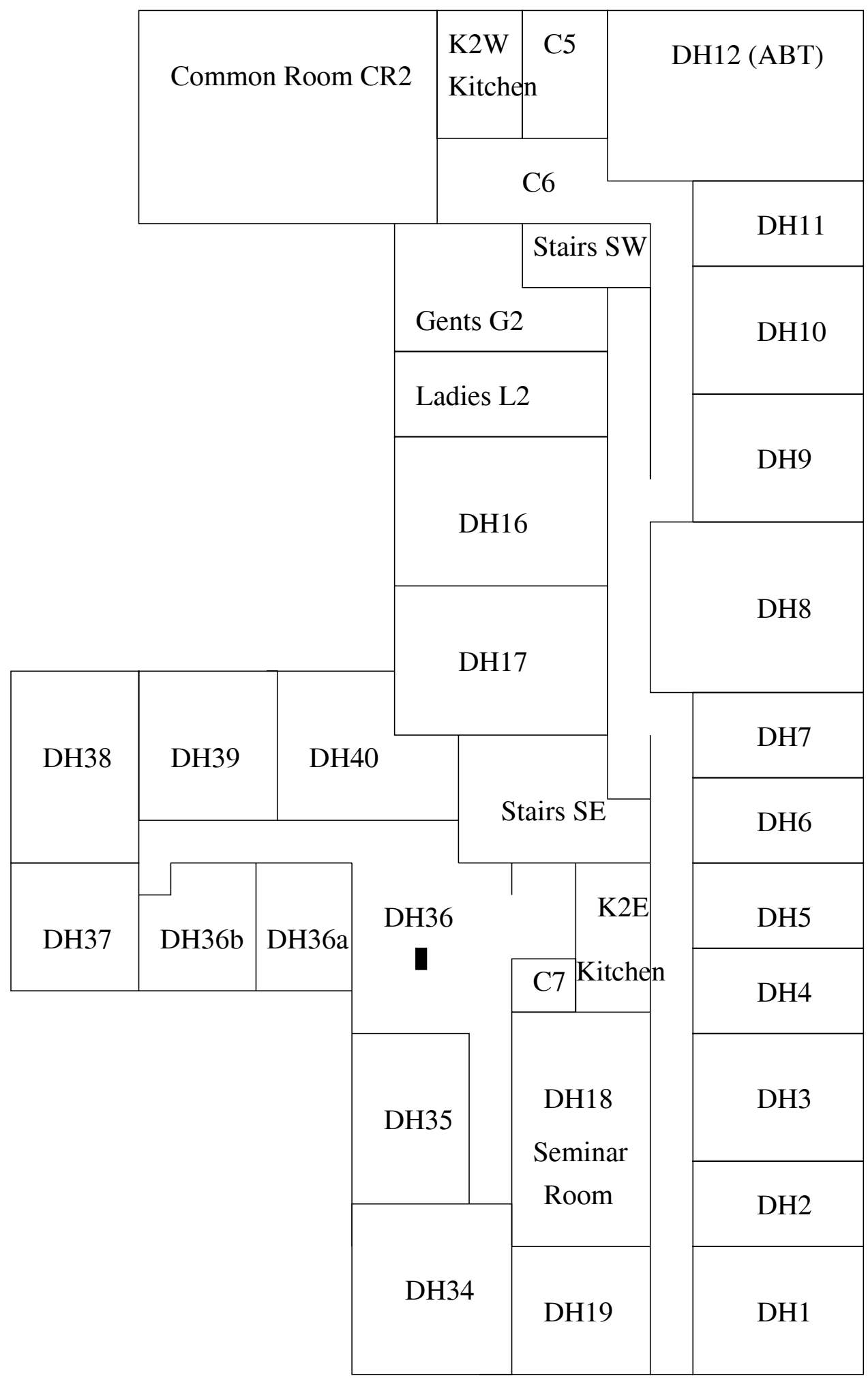

Figure 9: Floor plan of Dartington House 2nd floor. 


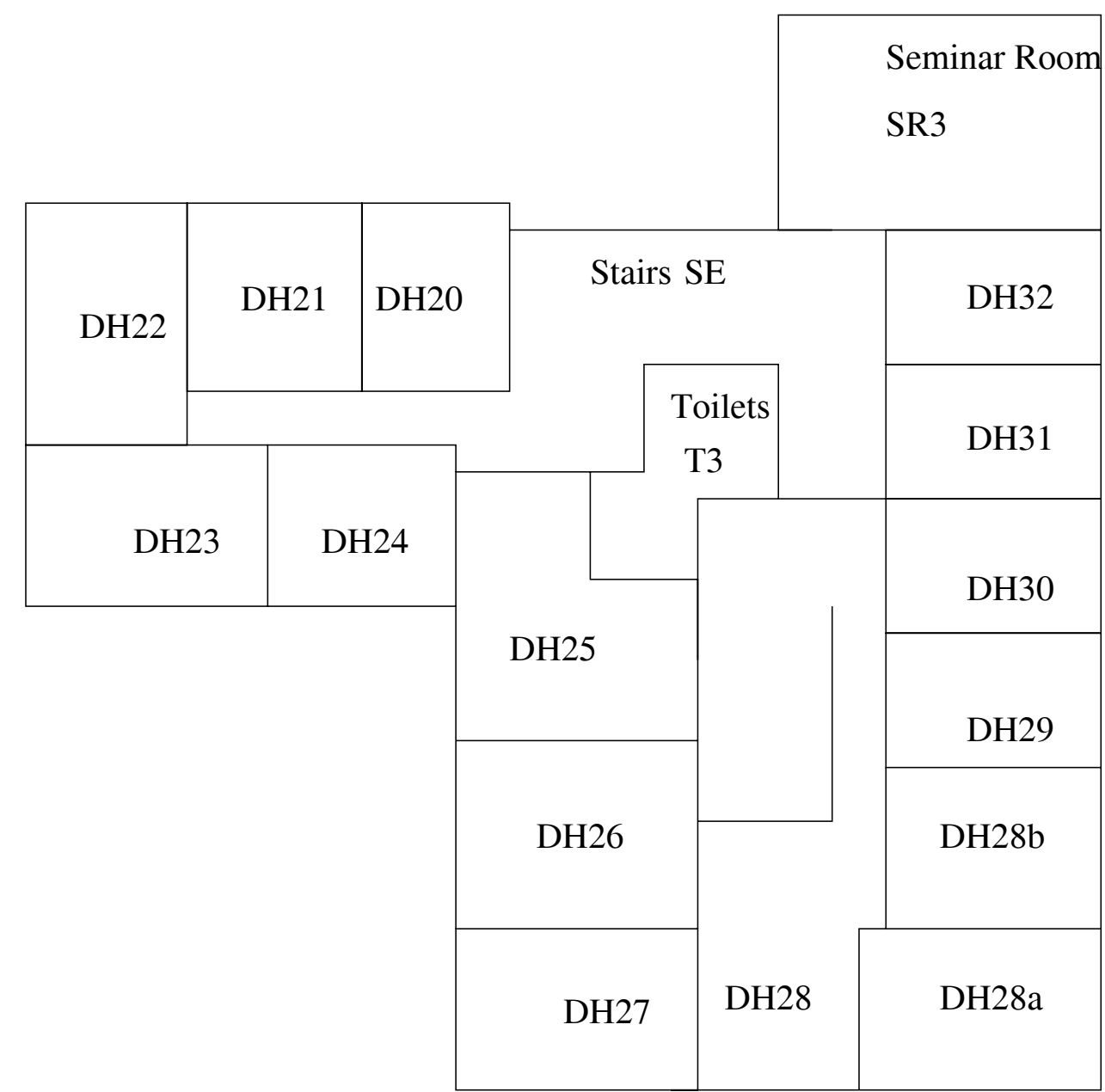

Figure 10: Floor plan of Dartington House 3rd floor.

\begin{tabular}{|l|l|}
\hline Colour & Rooms \\
\hline 1 & UOS1, C3, DH52, DH49a, DH47, DH45, C6, DH36 \\
\hline 2 & DH56, K1, C5, DH4, DH37, DH34, SR3, DH22, DH20, DH31, DH24, DH28 \\
\hline 3 & $\begin{array}{l}\text { UOS3, SW, L1, DH54, DH57, DH51, DH49b, C2, K2W, DH11, DH16 } \\
\text { DH18, DH3, DH1, DH40, DH35, DH32, DH23, T3, DH27 }\end{array}$ \\
\hline 4 & $\begin{array}{l}\text { UOS4, G1, DH55, SE, DH42, DH50, DH46, C4, DH43b, CR2, DH12, L2 } \\
\text { DH9, DH7, DH5, DH2, DH39, DH36a, C7, DH26, DH29, DH28a }\end{array}$ \\
\hline 5 & $\begin{array}{l}\text { UOS2, C1, DH53, DH43a, DH48, DH41, DH44, G2, DH10, DH8, DH17 } \\
\text { DH6, K2E, DH19, DH38, DH36b, DH21, DH25, DH30, DH28b }\end{array}$ \\
\hline
\end{tabular}

Table 1: A 5-colouring of the rooms in Dartington House. 


\section{$5 \quad$ Other issues}

There are various issues to do with electromagnetic diffraction that were raised by discussions on this problem but not addressed at the Study Group. We bear in mind that for frequencies of $2.5 \mathrm{GHz}$ and $5 \mathrm{GHz}$ the EM wavelengths are $12 \mathrm{~cm}$ and $6 \mathrm{~cm}$.
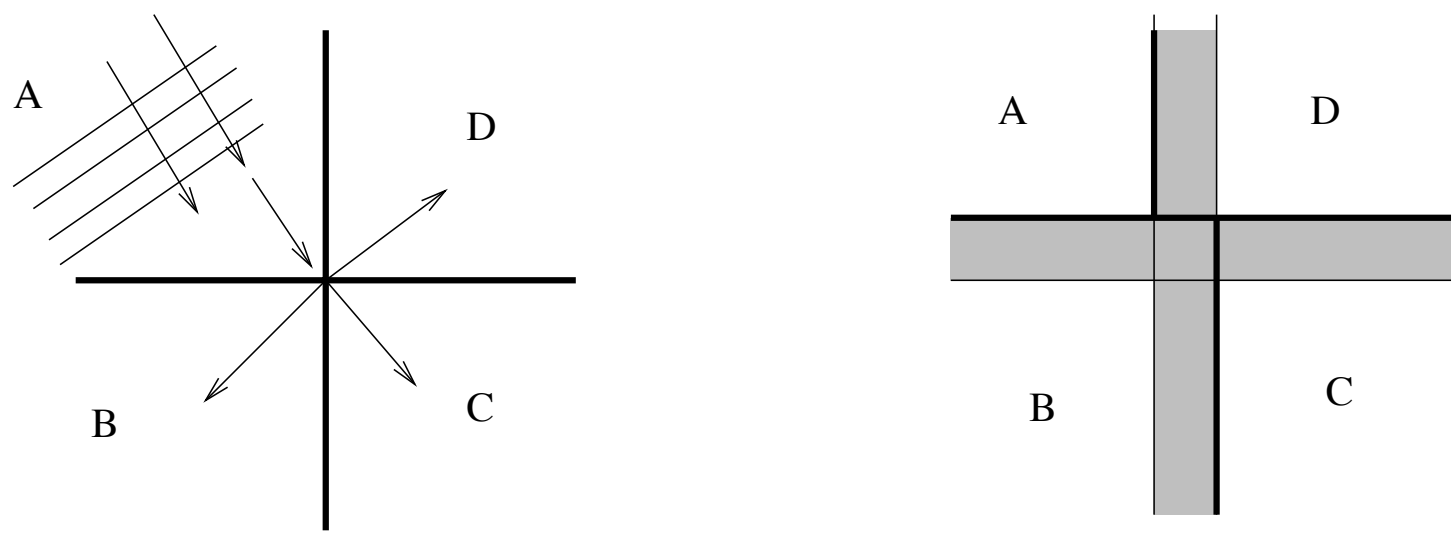

Figure 11: Diffraction at edges for rooms fitted with Stealthy Wallpaper, indicated by heavy lines.

(1) Diffraction at edges and corners. When 4 walls meet along a vertical edge as illustrated on the left in Figure 5, and an electromagnetic wave is incident from one room $A$, a diffraction problem has to be solved. Is the received field in the adjoining room $B$ dominated by the attenuated plane-wave transmission through the stealthy wallpaper on the wall between $A$ and $B$, or is it dominated by the diffracted field from the edge? Furthermore, is the received field in the diagonally opposite room $C$ dominated by the attenuated plane-wave transmission across 2 walls $(A \rightarrow B \rightarrow C$ and $A \rightarrow D \rightarrow C$ ) or by the diffracted field from the edge? This kind of question will depend on the detailed construction of the edge, and the fact that walls and floors may well be of thickness comparable to a wavelength, so that it matters which side of a wall Stealthy Wallpaper is fitted. For instance, if it is fitted as shown on the right of Figure 5 then although rooms $B$ and $D$ are diagonally opposite, they are only separated by one layer of SW. The accuracy of building construction may also play a part in these considerations.

(2) How significant is propagation through cracks and edges and corners generally, e.g. door frames and window frames? What coupling would be provided between one room and the next by a metal door handle - will it act as an aerial and effectively short circuit the insulation provided by Stealthy Wallpaper? What are the effects of other metallic objects that run through a building, e.g. mains wiring, phone lines, window frames, heating and air conditioning systems, plumbing etc?

Regrettably, there was no time to pursue these questions within the context of the Study Group. 


\section{Conclusions}

While we were unable to answer any of the specific questions asked in the proposal for this problem, good insights were made into the nature and solution of the practical problem. It is worthwhile to note here that there does not exist a simple upper bound for general problems of this nature and, while unlikely to appear in real cases, pathological cases can always be constructed.

While the above statement is true in general, we did find non-trivial upper bounds for a number of simplified cases which, although rare in real life, give an insight into practical steps which a building planner could take in order to arrange a building in a security conscious manner. Further, by taking into account the inherent order in real office buildings we were able to develop an heuristic algorithm allowing us to develop a colouring with a simple upper bound very quickly obtained from the plan itself. While not an optimal algorithm, it seems likely that the upper bound in most buildings would be below the maximum allowed count of frequencies for next generation wireless devices suggesting the algorithm has good potential application.

Overall, while not answering the more esoteric and mathematical questions posed in the initial proposal, we were able to develop a practical method for finding solutions to real problems. As such, we hope that this report will prove useful to BAE SYSTEMS.

We are grateful for conversations with Colin McDiarmid, Ross Kang, Andrew Thomason and Dominic Welsh and would like to thank Colin Sillence and BAE SYSTEMS for providing us with this interesting and thought-provoking question.

\section{A Code}

\section{A.1 Pseudo-code for the heuristic algorithm}

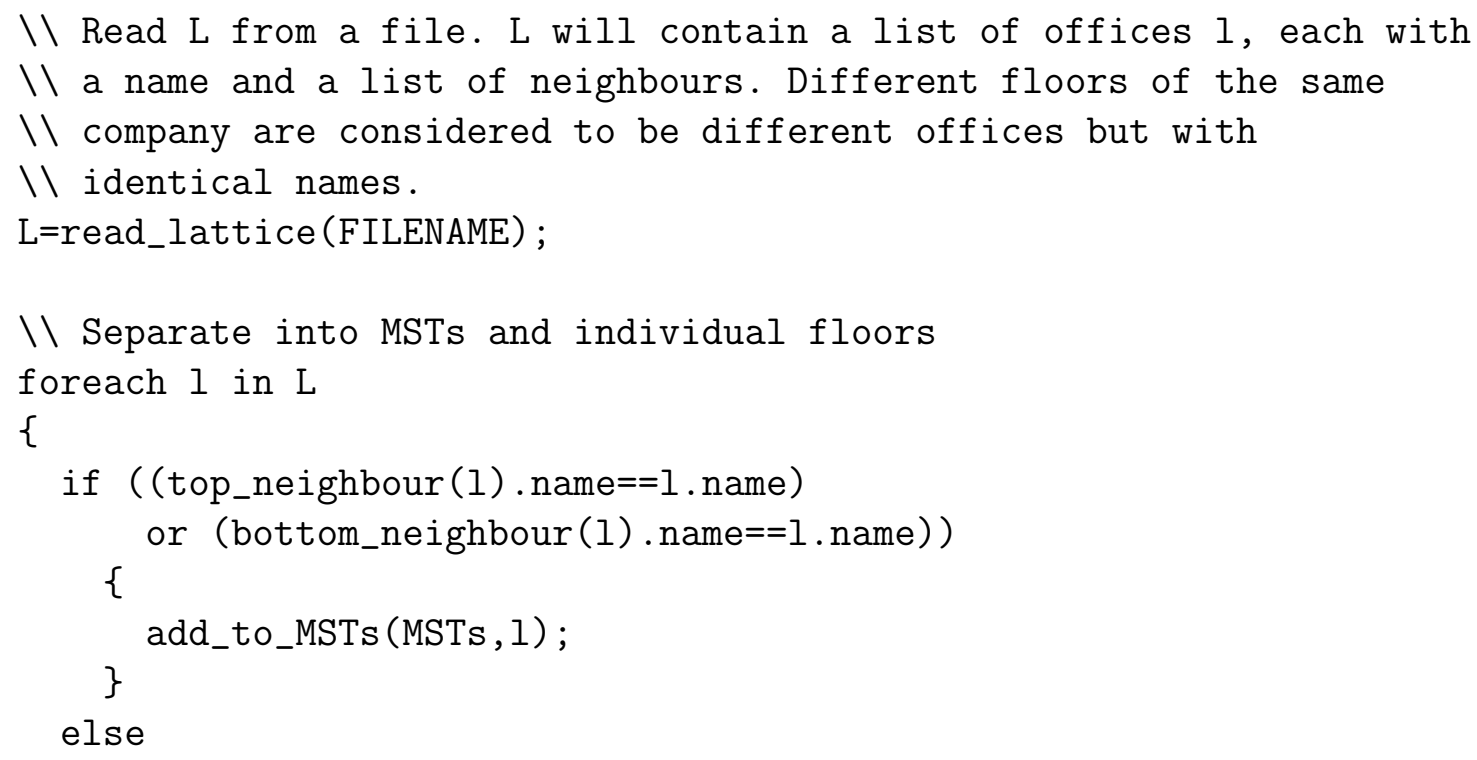




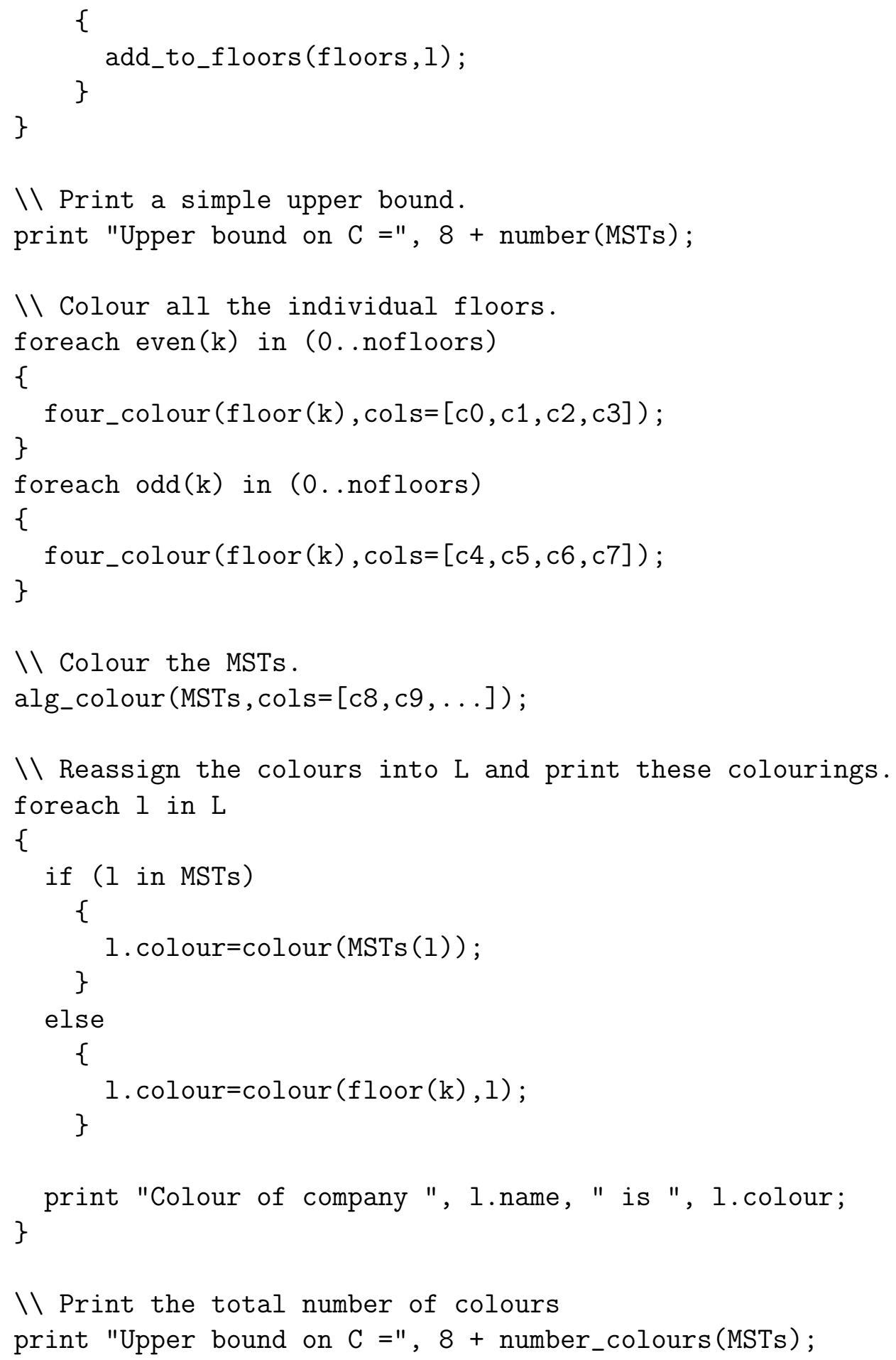

\section{A.2 Matlab code for exact colouring algorithm}

function chrom (neighbours)

$\%$ Chromatic number

$\%$ CHROM(name) uses the Brown-Brelaz-Peemoller algorithm to find the 
$\%$ chromatic number of the graph specified by the file name.txt. The $\%$ approach is a depth-first exhaustive search, which attempts to improve $\%$ upon a greedy initial assignment. Each new assignment that achieves a $\%$ smaller number of colour is displayed as it is found. Name.txt should $\%$ contain a space-separated list of neighbours for each node in turn, $\%$ with the neighbours for each node on a separate line.

$\%$ Open specified data file for reading

[fid,message $]=$ fopen (strcat (neighbours, '.txt'), ' $r$ ');

if $\mathrm{fid}==-1$

disp(message)

return

end

$\%$ Read lists of neighbours for each node in turn, using a strategy that

$\%$ pads the resulting matrix automatically with zeros, without generating

$\%$ any dimensioning errors.

$\mathrm{n}=0$;

while feof $($ fid $)==0$

$\mathrm{n}=\mathrm{n}+1$;

tline $=$ fgetl $(\mathrm{fid})$;

$f=\operatorname{strread}(t$ line);

$a(n, 1:$ length $(f))=f$;

end

fclose (fid);

$\%$ Check the validity of the extracted neighbour list

for $i=1: n$

$f=a(i, f i n d(a(i,:)>0))$;

for $j=1: \operatorname{length}(f)$

if isempty $(f$ ind $(a(f(j),:)==i))$

disp ('Error: invalid neighbour list')

return

end

end

end

$\%$ Start of main calculation: $\mathrm{k}$ is the current node for assignment,

$\%$ labs carries labels and $\mathrm{x}$ is the current assignment. An upper bound

$\%$ for the chromatic number is $q$.

back $=$ false; $k=1 ; \operatorname{labs}=\operatorname{zeros}(n, 1) ; x=\operatorname{zeros}(n, 1) ; q=n ;$

while true

if back $==$ true

$\mathrm{c}=\mathrm{x}(\mathrm{k}) ; \operatorname{labs}(\mathrm{k})=0$;

else

$c=0$;

end

$\%$ Look for lowest colour $y$ in $c+1, \ldots, q-1$ that is not

$\%$ currently used for a 
$\%$ neighbour $1,2, \ldots, \mathrm{k}-1$ of node $\mathrm{k}$;

$\%$ the colours that are currently used for

$\%$ these neighbours of node $k$ are extracted into the array $f$.

$\mathrm{f}=\mathrm{x}(\mathrm{a}(\mathrm{k}, \mathrm{f}$ ind $(\mathrm{a}(\mathrm{k},:)>0$ \& $\mathrm{a}(\mathrm{k},:)<\mathrm{k})))$;

$\mathrm{y}=0$;

for $z=c+1: q-1$

if isempty ( $f$ ind $(f==z)$ )

$\mathrm{y}=\mathrm{z}$;

break

end

end

$\%$ Move down one level in the colouring

if $\mathrm{y}>0$

$\mathrm{x}(\mathrm{k})=\mathrm{y}$;

$\mathrm{k}=\mathrm{k}+1$;

$\%$ When a new solution has been found then need to set $\mathrm{k}$ to the $\%$ minimal node rank with highest colour and remove labels from $\%$ nodes $\mathrm{k}, \ldots, \mathrm{n}$. Then set for backtracking.

if $(\mathrm{k}>\mathrm{n})$

$\mathrm{q}=\max (\max (\mathrm{x}))$;

disp(sprintf (['New solution using', ... \% $\%$ d with $\% 2 d$ highest instance(s)'], ...

$q$, length $($ find $(x==q)))$ )

$\mathrm{x}^{\prime}$

$\mathrm{k}=\min (\mathrm{find}(\mathrm{x}==\mathrm{q}))$;

$\operatorname{labs}(\mathrm{k}: \mathrm{n})=0$;

back = true;

else

back = false;

end

$\%$ Backtrack if we cannot move down to the next level

else

back = true;

end

$\%$ At this point, we have got stuck at level $\mathrm{k}$ and need to backtrack.

$\%$ Set $b$ to those nodes $1, \ldots, k-1$ that are adjacent to node $k$.

$\%$ Label any that have lowest rank among k's neighbours of the same

$\%$ colour. To do this, set $f$ to be the colours that are adjacent to $k$

$\%$ and then loop through them. (A possible improvement would be to

$\%$ remove any repeated values in $f$. )

if back $==$ true

$$
\begin{aligned}
& b=a(k, f \operatorname{ind}(a(k,:)>0 \& a(k,:)<k)) ; \\
& f=x(b) ; \\
& \text { for } i=1: \operatorname{length}(f) \\
& \quad l a b s(\min (b(f \operatorname{ind}(f==f(i)))))=1 ; \\
& \text { end }
\end{aligned}
$$




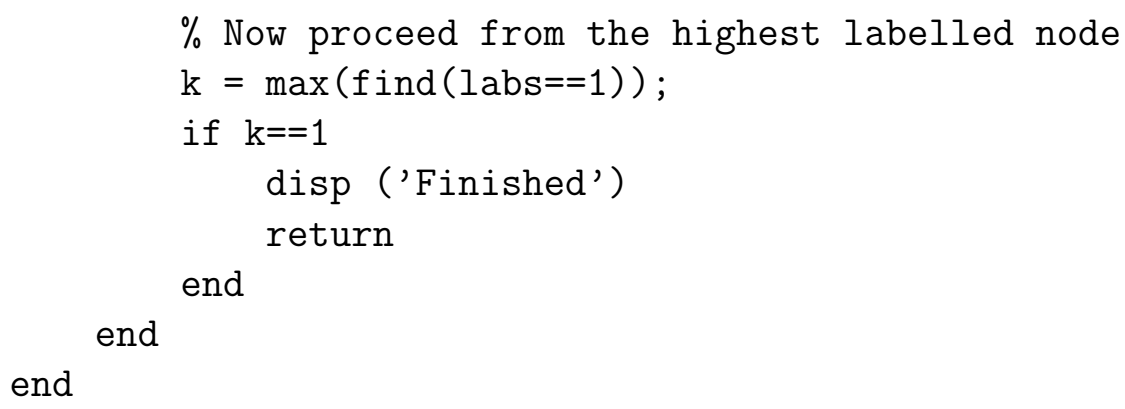

\section{References}

[1] H. Tietze. Famous Problems of Mathematics. Graylock, 1965.

[2] B. Bollobás. Graph Theory-An Introductory Course. Springer-Verlag, 1979.

[3] K. Appel and W. Haken. Every planar map is four colorable. Part I. Discharging. Illinois J. Math., pages 429-490, 1977.

[4] K. Appel and W. Haken. Every planar map is four colorable. Part II. Reducibility. Illinois J. Math., pages 491-567, 1977.

[5] N. Robertson, D. P. Sanders, P. D. Seymour, and R. Thomas. A new proof of the four colour theorem. Announc. Amer. Math. Soc. 2, pages 17-25 (electronic), 1996.

[6] H. Tietze. Über Nachbargebiete im Raume. Monatshefte für Mathematik und Physik, 16:211-216, 1905.

[7] A. S. Besicovitch. On Crum's Problem. Journal of the London Mathematical Society, pages 285-287, 1947.

[8] A. K. Dewdney and J. K. Vranch. A convex partitioning of $\mathbb{R}^{3}$ with applications to Crum's problem and Knuth's post-office problem. Utilitas Mathematica, 12:193-199, 1977.

[9] Joe Culberson. Graph colouring page - web resource.

URL: http://www.cs.ualberta.ca/ joe/Coloring/, 2004. 